\title{
A Viable Superluminal Hypothesis: Tachyon Emission from Orthopositronium
}

\author{
M. Skalsey ${ }^{1}$, R.S. Conti ${ }^{1}$, J.J. Engbrecht ${ }^{1}$, D.W. Gidley ${ }^{1}$, R.S. Vallery ${ }^{1}$, P. W. \\ Zitzewitz ${ }^{2}$ \\ ${ }^{I}$ Randall Laboratory of Physics, University of Michigan, Ann Arbor, MI \\ ${ }^{2}$ Department of Natural Sciences, University of Michigan: Dearborn, Dearborn, MI \\ (734)763-3464; skalsey@umich.edu
}

\begin{abstract}
Tachyons are hypothetical particles that travel faster than the vacuum speed of light. Previous experiments have searched for, but have not found evidence of tachyons. Long-standing, anomalous measurements of the orthopositronium (o-Ps) decay rate are interpreted as evidence for two tachyons being occasionally emitted when oPs decays. Restricting the coupling of tachyon pairs to a single photon (no tachyon coupling to matter) yields a new theory where tachyons are only observed in o-Ps decay and not in the previous tachyon experiments. Combining the single photon coupling theory with all previous experiments predicts that these tachyons must deposit energy while traversing scintillator detectors. A new tachyon search experiment will use this energy loss prediction to attempt to find tachyons passing through the apparatus or set limits disproving the original o-Ps to tachyon hypothesis. Viewing an intense o-Ps source, a time-of-flight spectrometer uses the superluminal property of tachyons for identification. Several months of continuous data acquisition will be necessary to completely eliminate the o-Ps to tachyon hypothesis.
\end{abstract}

\section{INTRODUCTION}

The velocity of light in vacuum, $\mathrm{c}$, is the ultimate speed for material objects. This limit follows from the theory of special relativity and is confirmed by observations. The possibility of accommodating faster-than-light particles into special relativity is a 40-year-old theoretical idea (Bilaniuk, 1962). The name tachyon (Feinberg, 1967), from Greek $\tau \alpha \chi \vee \zeta$ (swift or fast), is now applied to any proposed superluminal particle. Special relativity reveals nothing about the dynamical properties of tachyons, simply energy and momentum kinematics. Hence, for freely propagating tachyons, performing experimental searches are difficult and in recent years, have become unfashionable.

Previous tachyon experiments, primarily cosmic ray and accelerator searches, have set limits on tachyons and their interactions with ordinary matter (Hikasa, 1992). Most of these tachyon limits are several decades old. The current lack of interest stems from philosophical problems with freely propagating tachyons and causality. The principle of causality states, in the language of special relativity: For any given inertial observer, cause must chronologically precede its effect. Examples of how tachyons might violate causality have been published (Rolnick, 1969) and discussed extensively (Recami, 1986). Tachyon theories embracing causality [e.g. extended relativity (Recami,1986)] lead to no observable consequences for experimentation.

In spite of these philosophical barriers, a new tachyon search experiment is beginning based on the hypothesis that tachyons do not interact directly with ordinary matter, but just with light (photons). This new experiment has its origins in a long-standing anomaly in low energy atomic physics: the disagreement (Dobroliubov, 1993) between theory and experiment for the orthopositronium annihilation decay rate in vacuum $\left(\lambda_{T}\right)$. While the decay rate disagreement is small, $\Delta \lambda_{\mathrm{T}}{ }^{\text {exp-th }} / \lambda_{\mathrm{T}}=(0.14 \pm 0.02) \%$, it is statistically significant and also very important because the theoretical prediction comes from quantum electrodynamics (QED).

CP504, Space Technology and Applications International Forum-2000, edited by M. S. El-Genk

(C) 2000 American Institute of Physics 1-56396-919-X/00/\$17.00 
QED is the fundamental theory of the interaction of light and matter. The theoretical predictions of QED are enormously successful with numerous experimental verifications using many different systems and energy scales (Kinoshita, 1990). The lone, long-standing QED discrepancy is the $\lambda_{\mathrm{T}}$ problem. Orthopositronium (o-Ps) is the $\stackrel{\mathrm{f}}{S}=1$ (spin one) bound state of an electron and a positron (Rich,1981). QED predicts that, in vacuum, o-Ps annihilates into three photons with a lifetime of about $142 \mathrm{~ns}\left(\lambda_{\mathrm{T}}=1 / \tau\right)$. High precision $\lambda_{\mathrm{T}}$ measurements are $0.14 \%$ above the current QED theory.

Since $\lambda_{\mathrm{T}}{ }^{\mathrm{cxp}}>\lambda_{\mathrm{T}}{ }^{\text {th }}$, a possible explanation is that there is another decay mode for o-Ps, besides three photon ( $3 \gamma$ ), not included in the QED calculation. This idea is at least 20 years old (Mikaelian, 1978), but has never been applied to tachyons until now. In general, adding another non-3 $\gamma$ decay channel to o-Ps increases the observed decay rate, $\lambda^{\text {obs }}$, thusly:

$$
\lambda^{\text {obs }}=\lambda_{\mathrm{T}}+\lambda_{\text {new }}
$$

where $\lambda_{\text {new }}$ is the decay rate of o-Ps into the new channel. The $\lambda_{\mathrm{T}}$ experiments measure the total disappearance rate of o-Ps, regardless of decay products. Examples of $\lambda_{\text {new }}$ include normal processes like scattering, but also exotic particle or forbidden decays (Skalsey, 1997A). A branching ratio for decays into the new channel can be defined:

$$
\text { B.R. }=\lambda_{\text {new }} / \lambda^{\text {ohs }} \text {. }
$$

Notice only $0.14 \%$ of the o-Ps decays need to proceed through the new channel to obtain agreement between QED theory and $\lambda_{\mathrm{T}}$ experiments. Specifically, an o-Ps decay to a pair of tachyons is hypothesized:

$$
\mathrm{O}-\mathrm{Ps} \rightarrow \mathrm{T}+\overline{\mathrm{T}}
$$

where $T(\bar{T})$ is a tachyon (anti-tachyon or a second tachyon). The choice of a two-tachyon final state for the hypothesized decay is arbitrary. Numerous other possible final states have been hypothesized previously and many of these have been investigated experimentally (Skalsey, 1997A).

Directly coupling the tachyons to ordinary subluminal matter (o-Ps) is inconsistent with previous experiments. Existing limits and measurements preclude tachyon-matter interactions at a level necessary to explain the o-Ps $\lambda_{\mathrm{T}}$ discrepancy. Therefore, along with the hypothesized decay in Eqn. (3), a new theory of tachyon interactions is developed. This new theory couples the pair of tachyons to a single photon, no direct tachyon-matter interactions. The tachyon connection to o-Ps is established through a process called virtual, single photon annihilation of o-Ps, a unique property of positron-electron annihilation from the triplet $S=1$ state. This new theory of tachyons appears to be consistent with all available experimental information.

Conservation of momentum and energy applied to Eqn. (3) require back-to-back tachyon emission, each with an energy of $511 \mathrm{keV}$, the electron rest mass energy. The branching ratio for the decay in Eqn. (3) is taken to be $0.14 \%$ to match the $\lambda_{\mathrm{T}}$ problem. A sufficiently precise experiment, capable of detecting tachyons at this level, can therefore disprove this hypothesis. But, how does one detect tachyons without knowing their properties?

One approach, taken in early tachyon accelerator experiment (Baltay, 1970), is to look for missing energy and momentum in collision events. If a tachyon is produced in collision and then leaves the apparatus without interacting, an imbalance will appear in the final state products with a characteristic tachyon signature. The tachyon limits obtained from this early experiment (Baltay, 1970) are not sensitive enough to exclude the o-Ps to tachyon decay in Eqn. (3) (by a factor of roughly 100 to 1000). But this general technique has merit for experimental tachyon searches with o-Ps.

Consider an experiment with an o-Ps source surrounded by many photon detectors in all directions. If the detectors are thick enough, every time o-Ps decays, some photon energy must be observed. This type of set-up is referred to as a hermetic photon detector. Fortunately, this very difficult and expensive experiment has already been performed at the Univ. of Tokyo (Mitsui, 1993). The Tokyo experiment used one ton of scintillator material for the hermetic photon detector. Their result is a $3 \times 10^{-6}$ limit on the branching ratio of o-Ps to "invisible decays" (Mitsui, 1993), which are interpreted here as tachyon pair decays. 
To retain the o-Ps to tachyons hypothesis with $1.4 \times 10^{-3}$ branching ratio, the Univ. of Tokyo experiment must have somehow missed the tachyons that are assumed to leave their apparatus without interacting. If the tachyons had deposited some energy in their hermetic detector, the tachyons could have been overlooked in (Mitsui, 1993). The minimum deposited energy is about $300 \mathrm{keV}$ total or $150 \mathrm{keV}$ per tachyon. Combining the $150 \mathrm{keV}$ with the detector size, a range of energy deposition rates for tachyons is deduced. To summarize the conclusions, if the hypothetical decay o-Ps $\rightarrow T+\bar{T}$ exists with B.R. $=0.14 \%$, then the tachyons must deposit energy that can be observed in scintillator detectors.

Energy deposition by tachyons is the basis for the new tachyon search experiment. Tachyons are supplied by an intense o-Ps source. On one side of the source, a time-of-flight (TOF) spectrometer is being constructed to detect tachyons. The TOF of tachyons is shorter than any other correlated background traversing the apparatus, allowing unique identification. Limits on the tachyon branching ratio will be obtained in reasonable running times sufficient to disprove the o-Ps to tachyon explanation for the o-Ps $\lambda_{\mathrm{T}}$ problem.

\section{STATUS OF THE o-Ps DECAY RATE PROBLEM}

The decay rate, $\lambda=1 / \tau$, of o-Ps is expressed in QED as a series in $\alpha$, the fine structure constant $(\alpha \cong 1 / 137)$ :

$$
\lambda=\frac{4}{9}\left(\pi^{2}-9\right) \frac{\alpha^{6} m_{e} c^{2}}{h}\left[1+\mathrm{A} \frac{\alpha}{\pi}+\mathrm{B} \alpha \ln \alpha+\mathrm{C}\left(\frac{\alpha}{\pi}\right)^{2}+\ldots\right] .
$$

At present, the A and B coefficients are well known (Adkins, 1996; Karshenboim, 1993) as also some very small, even higher order terms. Without $C$, the theoretical result is $\lambda^{\text {th }}=7.03820 \mu \mathrm{s}^{-1}$. The $C$ coefficient is a very challenging calculation. At this time, only parts of the calculation have been published (Milstein, 1994; Burichenko, 1993; Labelle, 1994; Adkins, 1995A; Adkins, 1995B; Faustov, 1995). There is intense interest in finishing the C calculation (Adkins, 1998) because of the experimental situation.

Two recent experiments at the University of Michigan are both in significant disagreement with $\lambda^{\text {th }}$. The first (Westbrook, 1989) used low-density gases to form o-Ps and found $\lambda^{\text {gas }}=7.0514 \pm 0.0014 \mu \mathrm{s}^{-1}$. In subsequent investigations (Skalsey, 1998), we have found $\lambda^{\text {gas }}$ needs a small revision downward, but not down to theory. The second $\lambda$ experiment (Nico, 1990) used a slow $\mathrm{e}^{+}$beam $(<1 \mathrm{keV})$ to form o-Ps in an evacuated, MgO-lined cavity. This systematically different experiment yielded $\lambda^{\text {cavity }}=7.0482 \pm 0.0016 \mu \mathrm{s}^{-1}$, more than $6 \sigma$ from theory. The difference between theory and experiment $(\mathrm{Nico}, 1990)$ is $(0.14 \pm 0.02) \% . \lambda^{\text {cavity }}(\mathrm{Nico}, 1990)$ has withstood rigorous systematic testing (Gidley, 1991) and continues to be the major focus in our positronium program.

In 1995, a group at the Univ. of Tokyo published a new experimental $\lambda$ result (Asai, 1995), done in fine-grained $\mathrm{SiO}_{2}$ powders, and in agreement with theory: $\lambda^{\text {powd }}=7.0398 \pm 0.0029 \mu \mathrm{s}^{-1}$. Unfortunately, their analysis ignored the Stark effect on o-Ps. Large electric fields are known to be present in these insulating powders and the potential effect on $\lambda$ is discussed in detail in (Ford, 1976). The effects estimated in (Ford, 1976) are large compared to the error bar of (Asai, 1995) and correcting will raise their decay rate value. This shift can be understood classically as the electric field pulling the $\mathrm{e}^{+}$and $\mathrm{e}^{-}$farther apart, reducing the overlap and the annihilation rate. The quantum mechanical argument involving P states is given in (Skalsey, 1997A). We have discussed the Stark effect with the head of the Tokyo group, Prof. T. Hyodo and they now agree that the Stark effect must be accounted for (Hyodo, 1997). As an aside, the parapositronium [p-Ps: $\mathrm{e}^{+} \mathrm{e}^{-}$spin zero ground state] decay rate has just recently been calculated including all the $\alpha^{2}$ correction terms (Czarnecki,1999). Measurements of the p-Ps decay rate (AlRamadhan, 1994) done at the Univ. of Mich. are in excellent agreement with theory, and the fractional experimental uncertainty is the same as the o-Ps studies, $0.02 \%$. This somewhat surprising result is consistent with the o-Ps tachyon decay hypothesis.

The o-Ps decay rate remains a serious problem for QED. Most of our positronium program is dedicated to checking and rechecking our decay rate results. The theorists are busy finishing the $\mathrm{C}$ calculations, but no really big, resolvethe-discrepancy terms have been found yet. Further the convergence of the QED series has recently been questioned (Adkins, 1998), leaving future resolution of this problem an open question. We, as experimentalists can only check our apparatus and then search for other, non-QED explanations, like tachyons. 


\section{PROPOSED o-Ps $\rightarrow$ T $\bar{T}$}

The proposed decay branch to be considered here is o-Ps $\rightarrow T+\bar{T}$ (T-tachyon, $\bar{T}$-antitachyon or a second tachyon), occurring at $1.4 \times 10^{-3}$ of the dominant rate, o-Ps $\rightarrow 3 \gamma$. Since o-Ps decays essentially at rest in the laboratory, the $\mathrm{T} \overline{\mathrm{T}}$ products are emitted back-to-back, each with a total energy of $511 \mathrm{keV}\left(=\mathrm{m}_{\mathrm{e}} \mathrm{c}^{2}\right)$. To obtain these results, conservation of momentum and energy are assumed during the decay. Tachyons with real energies and momenta can be included in special relativity if tachyons possess an imaginary rest mass: $\mathrm{M}=i \mu, \mu$ - positive real and called proper mass [see Feinberg's Scientific American article (Feinberg, 1970)]. Kinematics results, like equal energy, back-to-back tachyon emission from o-Ps, can then be derived. Off hand it may seem likely that such a simple decay should have been seen in some previous experiment. It will be shown that this is not the case. The special properties of o-Ps give the proposed tachyon decay unique characteristics that set it apart from all previous tachyon searches and simultaneously maintain consistency with high precision tests of QED. A useful property of o-Ps is called single photon virtual annihilation, an effect that is known to occur in QED as a radiative correction term. The particular use for this situation is shown in the Feynman diagram in Fig.lc depicting o-Ps decaying into a single photon that decays into two tachyons. [This type of use of single photon virtual annihilation is not unprecedented. It was used in discussing a hypothesized charge conjugation odd boson participating in o-Ps decay (Skalsey, 1997B) and S. Glashow used it in discussing possible mirror universes (Glashow, 1986; Gninenko, 1994)].

Charge conjugation invariance requires $\mathrm{e}^{+} \mathrm{e}^{-}$annihilation from the singlet state (e.g. p-Ps) coupling to an even number of photons (usually two Fig.1a). Conversely, triplet states (e.g. o-Ps) couple to an odd number of photons, real decays require $3 \gamma$ (Fig.1b) to conserve momentum and energy or virtual decays to $1 \gamma$ (Fig.1c, Fig.1d will be discussed later.) This even/odd property makes other QED tests less sensitive to tachyons since they would appear only in higher order correction terms and, given their inherently weak coupling, render them undetectable. While it is not possible to prove the previous statement for all cases here (there are numerous, high precision QED tests and each must be addressed), the example of the electron g-factor will be roughly worked out. A crude scaling scheme labels each electron-photon vertex with amplitude e and squaring gives the rate $\alpha$, the fine structure constant, roughly $10^{-2}$. In Fig. $1 \mathrm{~b}$, there are three such vertices giving $10^{-6}$. If Fig. $1 \mathrm{c}$ is to have a rate of $10^{-3}$ compared to Fig. $1 \mathrm{~b}$, then an overall rate of $10^{-9}$ is estimated for Fig. 1c. Since there is only one e- $\gamma$ vertex in Fig. 1c, the tachyon vertex corresponds to a $10^{-7}$ rate. To insert this vertex into the electron g-factor, a closed tachyon loop with two $10^{-7}$ vertices is the lowest order contribution. This loop can be put in the g-factor diagram itself or the lowest order anomaly diagram. Either way the tachyon contribution to the electron g-factor is at least 100 times smaller than the present experimental sensitivity, making the tachyon component negligible.

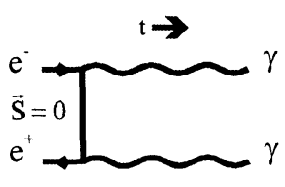

a) Single state, parapositronium and direct $\mathrm{e}^{+}-\mathrm{e}^{-}$annihilation.

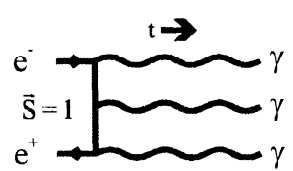

b) Triplet state, orthopositronium

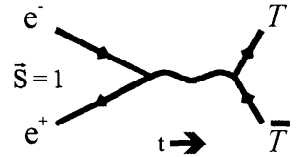

c) The decay o-Ps $\mathrm{z} T+\bar{T}$.

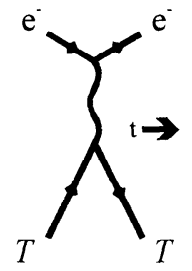

d) Rotating c) implies tachyons interact weakly with matter.

FIGURE 1. Feynman Diagrams for normal QED decays [ a) and b)] and processes with tachyons [ c) and d)].

The same conclusion pertains to cosmic-ray searches for tachyons. Positronium, binding energy $6.8 \mathrm{eV}$, is exclusively a low energy phenomenon. In cosmic-ray showers, $\mathrm{e}^{+}$annihilation-in-flight (Kantele, 1973) is the pertinent process, which is predominately (99\%) $2 \gamma$ annihilation from the singlet state. The quote from (Kantele, 1973): "the three-quantum annihilation can always be neglected" overstates the situation but shows the $3 \gamma$ component from the triplet state is small. High-energy accelerator experiments also find a very few $\mathrm{e}^{+} \mathrm{e}^{-} \rightarrow 3 \gamma$ events at 30 and $80 \mathrm{GeV}$ and the rate agrees with QED theory when detector thresholds are properly accounted for (Fernandez, 1987; Martyn, 1990; L3 Collab, 1990; Opal Collab, 1991). The complication of detector threshold sensitivity combined with the complexity of cosmic-ray showers precludes a quantitative analysis of triplet state $\mathrm{e}^{+} \mathrm{e}^{-}$ annihilation rates in the previous tachyon cosmic-ray searches (Marini, 1982; Bhat, 1979; Bartlett, 1978; Smith, 
1977; Prescott, 1976; Hazen, 1975; Clay, 1974). However, we have discussed this problem with Prof. Wayne Hazen (Hazen, 1975) who explained that these experiments were not analyzed for the fundamental processes (as we desire) but rather, to simply disprove the Clay and Crouch (Clay, 1974) positive tachyon result. Prof. Hazen's feeling was that the previous tachyon cosmic-ray searches lacked the sensitivity to observe tachyons from triplet state $\mathrm{e}^{+} \mathrm{e}^{-}$ annihilations-in-flight. Therefore previous cosmic-ray tachyon searches do not seem to exclude our proposed tachyon experiments, which will also attempt to directly detect the tachyons.

Direct tachyon detection is a formidable task considering nothing is known about tachyons except superluminal speed. The alternate approach, pioneered by Feinberg (Baltray, 1970), produces the hypothesized tachyon in a collision between a high-energy proton and an atom in a bubble chamber. The collision products are analyzed event-by-event, searching for missing momentum and energy due to a tachyon departing the apparatus undetected. This scheme makes very mild assumptions about tachyon interactions in detectors, unfortunately, the limits are not very restrictive. An equivalent o-Ps minimal assumption experiment (Mitsui, 1993) has already been performed, searching for o-Ps $\rightarrow$ "nothing", i.e. undetected decay products, which are interpreted here as tachyons. This monstrous and expensive experiment used about one ton of CsI and $\mathrm{NaI}$ scintillator detectors surrounding a welltagged o-Ps source. A limit of $2.8 \times 10^{-6}$ at $90 \%$ confidence was thereby derived on the branching ratio for o-Ps decays to undetectables (Mitsui, 1993) disproving that, if tachyons are produced, then they leave the apparatus without interacting. This very important result implies that if the decay o-Ps $\rightarrow T+\bar{T}$ exists, the tachyons deposit energy while leaving their apparatus. The deduced conclusion is that the tachyons must lose energy in traversing scintillator detectors, a conclusion that is actually not surprising. The original hypothesis, that o-Ps $\rightarrow T+\bar{T}$ exists through a presumed coupling, predicts that tachyons will interact with detectors through the same coupling. Fig. 1d is a rotated version of Fig. 1c with the same couplings and displays a propagating tachyon interacting with an clectron. The interpretation is that propagating tachyons infrequently strike electrons and ionize atoms, the principle of scintillation detection. Tachyons traversing detectors would lose at least some of their energy, probably as a long weak ionization trail. The rules for calculations from rotating Feynman diagrams that involve tachyons are unclear at this time. So one should not expect quantitative agreement between this tachyon theory and (Mitsui, 1993). The energy loss units measure essentially the number of electrons, no material dependence will be assumed. Normal charged particles traversing material roughly follow this simple approximation. The tachyon stopping power assumption could be criticized thusly: Suppose all or most of the tachyon energy is lost in a single interaction (photons frequently interact with matter in this manner.) Another tachyon decay model for o-Ps can be constructed with only one tachyon and one photon in the final state. In this alternative model, coupling between the initial state(o-Ps) and the final state (tachyon and photon) is through the same virtual single photon annihilation as with the two-tachyon final state theory. The one-tachyon theory predicts full energy deposition in a local region. Previous oPs experiments place restrictions on these tachyons (Gidley, 1991), leaving the stopping power assumption as the most plausible. Details of the analysis and limits for the single tachyon theory will be published elsewhere.

The o-Ps $\rightarrow$ "nothing" experiment (Mitsui, 1993) places a lower limit on the stopping power of tachyons $\mathrm{S}_{\mathrm{T}}>1 \mathrm{keV}-\mathrm{cm}^{2} / \mathrm{g}$, i.e. values smaller than this are excluded by (Mitsui, 1993). Further, an upper limit of $\mathrm{S}_{\mathrm{T}}<$ $500 \mathrm{keV}-\mathrm{cm}^{2} / \mathrm{g}$ is also established from (Mitsui, 1993). If tachyons lose energy too fast, they do not escape the source region. The upper limit of $500 \mathrm{keV}-\mathrm{cm}^{2} / \mathrm{g}$ is only a factor of two smaller than minimum ionizing particles (e.g. a low energy muon). These stopping power limits are the starting point for our proposed experiments, which will survey $S_{\mathrm{T}}$ values in the primary range $1-500 \mathrm{keV}-\mathrm{cm}^{2} / \mathrm{g}$ and, if possible, higher values.

\section{PROPOSED EXPERIMENTS}

Figure 2 shows the experimental time-of-flight (TOF) set-up to investigate the lower range of $\mathrm{S}_{\mathrm{T}}$ values. The major components are detectors and a thin ${ }^{64} \mathrm{Cu} \mathrm{e}{ }^{+}$source, with activity of up to $2 \mathrm{Ci}$ and a half-life of $12.6 \mathrm{hr}$. Activation of ${ }^{64} \mathrm{Cu} \mathrm{e}^{+}$sources in the Ford Nuclear Reactor on the Univ. of Mich., campus uses a maximum in-core thermal neutron flux of $3 \times 10^{13} \mathrm{n} / \mathrm{cm}^{2}$-sec. A thin, natural $\mathrm{Cu}$ foil is held in a polyethylene tube while in the reactor. The thin $\mathrm{e}^{+}$source thus produced is placed between two compressed pellets of fine-grained $\mathrm{MgO}$ powder, where about $20-25 \%$ of the $\mathrm{e}^{+}$stop and form positronium. Paramagnetic $\mathrm{O}_{2}$, which rapidly quenches o-Ps (Kakimoto, 1990) is excluded from the source $\mathrm{MgO}$ region by using a pure $\mathrm{N}_{2}$ atmosphere. With the $\mathrm{N}_{2}$ pressure just above latm, thin plastic walls can contain the source foil and $\mathrm{MgO}$ pellets. Making the o-Ps source region as massless as possible is 
important for investigating the larger values of $\mathrm{S}_{\mathrm{T}}: 100 \mathrm{keV}-\mathrm{cm}^{2} / \mathrm{g}<\mathrm{S}_{\mathrm{T}}<500 \mathrm{keV}-\mathrm{cm}^{2} / \mathrm{g}$, so the tachyon can get out of the source and deposit energy in the detectors. The large $S_{\mathrm{T}}$ experiment is shown in Fig. 3 . Note, the detectors are much thinner than those in Fig. 2, the thickness being only $3.5 \mathrm{~mm}$.

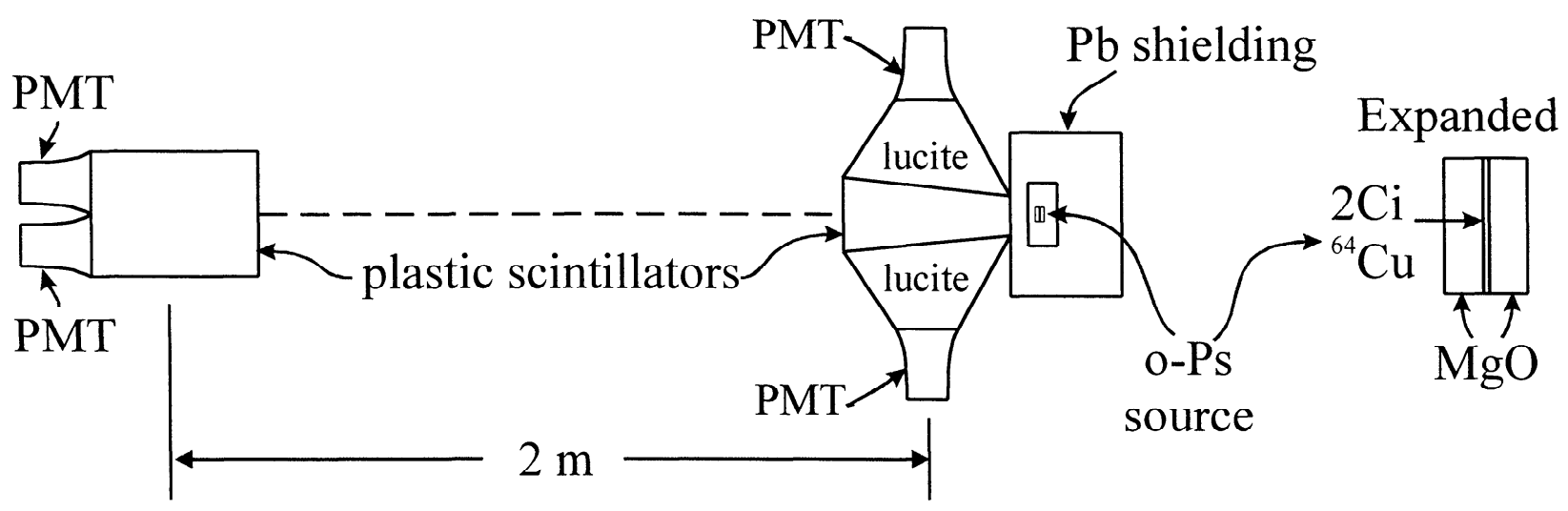

FIGURE 2. Tachyon TOF experiment using thick detectors and large activity sources.

From solid angle constraints in Fig. 2, the second, further-from-the-source, detector is much larger in volume than the close detector. The large size scintillators need several 4" photomultipliers on each to obtain good light collection efficiency. For large pieces of scintillator, the timing resolution is degraded simply by the large spatial extent of the detector and the finite speed of light. For smaller detector volumes, better time resolution can be obtained and faster $2 "$ phototubes are appropriate.

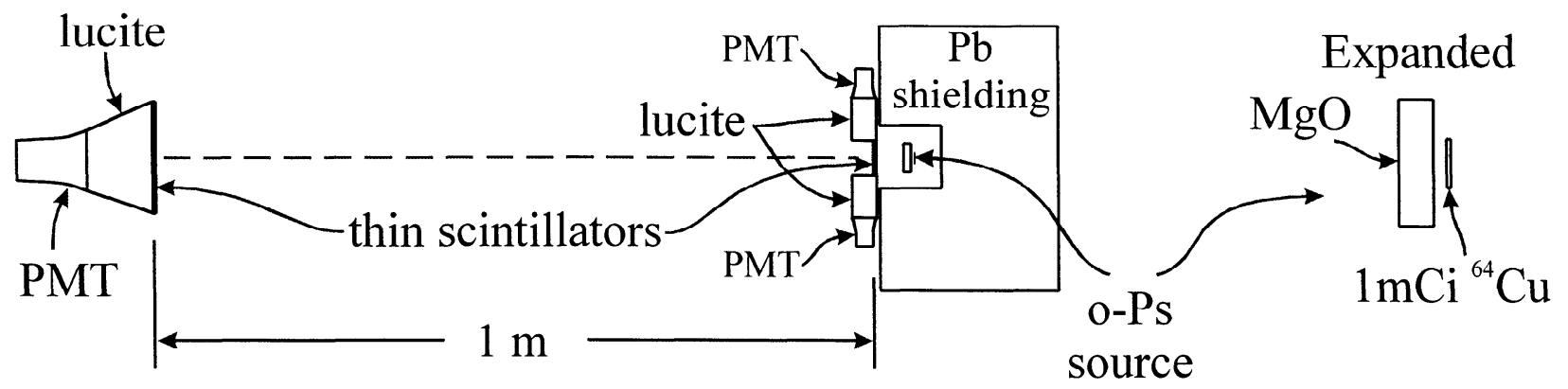

FIGURE 3. Another tachyon TOF experiment using thin detectors and low activity sources.

The important points in obtaining fast time resolution from plastic scintillator detectors are 1) good light collection efficiency, 2) good phototubes and, finally 3) signal processing with constant fraction timing discriminators. Constant fraction refers to timing derived in such a way as to be independent of the signal pulse heights, which span a wide range in plastic scintillator detectors. The timing signals from the discriminators are fed to a high-rate, timeto-digital converter (CAMAC-LeCroy 4204) interfaced to a PC computer, which records time spectra. The time resolution for the best detectors is expected to be $0.5 \mathrm{~ns}$ FWHM. The time resolution for the largest detectors will be a real concern if greater than 3 ns FWHM; sensitivity to tachyons could be severely reduced. To compensate for bad time resolution, the flight path between the detectors would need to be increased, reducing the solid angle roughly as the square of that increase.

One final correction must be considered, i.e. the scattering angle of tachyons when depositing energy. In normal multiple or plural scattering theory, an average or r.m.s. scattering angle is calculated after many collisions. It can be shown that larger tachyon masses will deflect less for a given energy deposition. Without more knowledge of tachyon dynamics, only a crude estimate can be made of how many tachyons will scatter enough in the first detector to miss the second detector, a conservative estimate is $90 \%$ will miss, i.e. a factor of ten loss in event rate. In Table $\mathrm{I}$, tachyon event rates are calculated for the lowest and highest $\mathrm{S}_{\mathrm{T}}$ configurations shown in Fig 2 and Fig 3 , 
respectively. Two more configurations with sizes between the two shown here are also required to complete the experiment, but they are omitted from Table I. The detected tachyon event rate is expressed in terms of the scintillator detector efficiency $\varepsilon$. With hundreds of $\mathrm{keV}$ deposited energy, (also estimated in Table 1) $\varepsilon$ will approach unity. At $40 \mathrm{keV}$ deposited energy, however, $\varepsilon$ will be quite diminished, to probably about 0.1 . Tachyon event rates in the range $0.004-4 \mathrm{~s}^{-1}$ are therefore predicted, taking $\varepsilon^{2}=0.01$. From these numbers, one concludes that much longer running times are required with weak sources. Extra small sources can be activated at the outside reactor surface and retrieved without turning the reactor off, greatly increasing the small source running time.

TABLE 1. Estimates of Signal and Noise Event Rates and Energy Loss.

\begin{tabular}{|c|c|c|c|c|}
\hline Figure 2 Setup & \multirow{4}{*}{\multicolumn{3}{|c|}{ Specification }} & Figure 3 Setup \\
\hline $\mathrm{S}_{\mathrm{T}}$ between & & & & $\mathrm{S}_{\mathrm{T}}$ between \\
\hline $1-4 \mathrm{keV}-\mathrm{cm}^{2}$ & & & & $100-500 \mathrm{keV}-\mathrm{cm}^{2}$ \\
\hline $\mathrm{g}$ & & & & (10 \\
\hline $\begin{array}{c}7.4 \times 10^{10} \frac{\text { decays }}{\mathrm{s}} \\
(2 \mathrm{Ci})\end{array}$ & \multicolumn{3}{|c|}{ source activity } & $\begin{array}{c}3.7 \times 10^{7} \frac{\text { decays }}{\mathrm{s}} \\
(1 \mathrm{mCi})\end{array}$ \\
\hline $1.41 \times 10^{10} \frac{\mathrm{e}^{+}}{\mathrm{s}}$ & $19 \%$ & positron fraction from source & $19 \%$ & $7.0 \times 10^{6} \frac{\mathrm{e}^{+}}{\mathrm{s}}$ \\
\hline $8.4 \times 10^{9} \frac{\mathrm{e}^{+}}{\mathrm{s}}$ & $60 \%$ & positrons entering $\mathrm{MgO}$ & $30 \%$ & $2.1 \times 10^{6} \frac{\mathrm{e}^{+}}{\mathrm{s}}$ \\
\hline $1.27 \times 10^{9} \frac{\mathrm{o}-\mathrm{Ps}}{\mathrm{s}}$ & $15 \%$ & positrons forming o-Ps & $15 \%$ & $3.2 \times 10^{6} \frac{\mathrm{o}-\mathrm{Ps}}{\mathrm{s}}$ \\
\hline $2.0 \times 10^{6} \frac{T \bar{T}}{\mathrm{~s}}$ & $1.6 \times 10^{-3}$ & o-Ps decaying into $T+\bar{T}$ & $1.6 \times 10^{-3}$ & $500 \frac{T \bar{T}}{\mathrm{~s}}$ \\
\hline $400 \frac{\text { incident } T}{s}$ & $4 \times 10^{-3}$ & Tacyons entering detectors & $8 \times 10^{-4}$ & $0.4 \frac{\text { incident } T}{s}$ \\
\hline $400 \varepsilon^{2} \frac{\text { detected } T}{s}$ & \multicolumn{3}{|c|}{ Tacyons detected ( $\varepsilon$ - detector efficiency) } & $0.4 \varepsilon^{2} \frac{\text { detected } T}{s}$ \\
\hline $0.25 \mathrm{~s}^{-1} \& 4 \mathrm{~s}^{-1}$ & \multicolumn{3}{|c|}{ uncorrelated and correlated noise rates } & $0.014 \mathrm{~s}^{-1} \& 0.9 \mathrm{~s}^{-1}$ \\
\hline negligible & \multicolumn{3}{|c|}{ Tachyon energy deposited in source } & $20-100 \mathrm{keV}$ \\
\hline $60-240 \mathrm{keV}$ & \multicolumn{3}{|c|}{ Tachyon energy deposited in $\mathrm{Pb}$} & 0 \\
\hline $40-160 \mathrm{keV}$ & \multicolumn{3}{|c|}{ Tachyon energy deposited in detector 1} & $40-200 \mathrm{keV}$ \\
\hline $40-111 \mathrm{keV}$ & \multicolumn{3}{|c|}{ Tachyon energy deposited in detector 2} & $40-200 \mathrm{keV}$ \\
\hline $0.2-0.8 \mathrm{keV}$ & \multicolumn{3}{|c|}{ Tachyon energy deposited in air } & $10-50 \mathrm{keV}$ \\
\hline
\end{tabular}

The final issue concerns predicting noise rates, correlated and uncorrelated, in the tachyon TOF spectrum. The most prevalent correlated noise source will be a single $\gamma$-ray Compton scattering in the first TOF detector and then the scattered $\gamma$-ray detected in the second detector. Of course, the transit time will correspond to $\mathrm{v}=\mathrm{c}$. All sources of correlated noise that have been considered result in $\mathrm{v} \leq \mathrm{c}$, with the exception of $\mathrm{t}<\mathrm{o}$ events e.g. cosmic ray muons going backwards through the apparatus. This muon noise can be measured with no source present. Any tachyon signal $(\mathrm{v}>\mathrm{c})$ should have a different transit time than any correlated noise source. The necessary time resolution (FWHM: 0.5 to $2.5 \mathrm{~ns}$ ) of the TOF system has been discussed previously. Separating the tachyon signal from correlated noise will be a strong function of the tachyon velocity, and consequently the assumed mass. Tachyons with large proper masses will be the easiest to set limits on, the limits will worsen as $\mathrm{m}_{\mathrm{T}} \rightarrow \mathrm{im}_{\mathrm{e}}$. Uncorrelated noise in a TOF spectrum can arise when, say, two $\gamma$-rays from two, almost simultaneous decays fire the detectors. Since there is no time correlation in such an event, it leads to a relatively flat background over the entire time spectrum. Any tachyon peak, separated from the correlated noise peak, will still be situated on top of this uncorrelated background. Using estimated $\gamma$-ray rates in the detectors, the uncorrelated noise rate has been calculated for a peaksized time window. The results are shown in Table I along with correlated noise rate estimates derived by considering Compton scattering only, the dominant correlated noise source. The choice of the ${ }^{64} \mathrm{Cu}$ positron source maximizes the signal to noise ratio in this experiment. 
From the estimates of correlated noise rates, it is clear that the time resolution of the TOF system must be smaller (a factor of three is used here) than the photon flight time to separate the tachyon and photon time peaks. Hence, the observed time resolution in the largest detectors will determine the final, longest flight path distance, assumed now to be $2 \mathrm{~m}$. The correlated noise will limit the experimental sensitivity as the assumed tachyon mass becomes as small as $i_{\mathrm{e}}$. For larger masses, the uncorrelated noise (flat background) in the time spectrum will limit the tachyon sensitivity. In the large mass limit, the sensitivity to small $\mathrm{S}_{\mathrm{T}}$ tachyons (Table Ia) will enable branching ratio limits of better than $10^{-5}$, assuming ten of the hottest (2Ci) irradiations, giving about 5 days of running with $>1 \mathrm{Ci}$. Again in the large tachyon mass limit, large $\mathrm{S}_{\mathrm{T}}$ tachyons (Table $\mathrm{Ib}$ ) will have branching ratio of limits of $10^{-1}$ from 100 days of running many $1 \mathrm{mCi}$ sources. To exclude tachyons from causing the o-Ps decay rate discrepancy, branching ratio limits of $10^{-4}$ are sufficient. Naturally, if a positive tachyon signal were observed, systematic tests of the result would be performed. Examples of these systematic tests are: 1) change the flight path between the detectors using as wide a variation in detector size as possible. 2) change the $\mathrm{MgO}$ powder to a $\mathrm{SiO}_{2}$ powder with similar properties or other Ps formation media like gases. 3) change the processing and data acquisition electronics, etc. Modifications and additions to this apparatus could investigate tachyon properties, if observed.

At this point, it is important to restate the required assumptions for this investigation: 1) Relativistic energy and momentum are conserved; 2) The exotic decay branch o-Ps $\rightarrow \mathrm{T} \overline{\mathrm{T}}$ occurs at the $1.4 \times 10^{-3}$ level, from assumption 1 , yielding back-to-back two tachyon emission, each starting with $511 \mathrm{keV}$ energy. Notice that the conclusion that these tachyons deposit energy in scintillator is not an assumption, this is a deduced fact from the previous o-Ps $\rightarrow$ "nothing" experiment. However, further assumptions are still required about the energy deposition process, i.e., it is uniform and does not greatly deflect the tachyons.

\section{CONCLUSIONS}

To conclude, a discrepancy between QED theory and an atomic physics experiment is interpreted as indirect evidence for tachyon emission. The hypothesized tachyon emission process is analyzed and found to be consistent with (i.e. not excluded by) previous experiments. An existing search for direct evidence of the tachyon emission process implies that a tachyon, if it exists, deposits measurable amounts of energy in scintillator detectors. Using this fact, an experiment has been designed to observe tachyons moving faster than the speed of light through an apparatus. A real, positive result would be a major breakthrough. More likely, a negative result, no tachyons or other anomalies observed, will be sufficiently precise to address the atomic physics discrepancy over a wide range of possible tachyon masses. In the large mass limit, all possible stopping powers will be excluded and the original hypothesis is discredited. In the unlikely event that tachyons were discovered, many questions would surface and more experimentation would follow. The relation of tachyons to spacecraft propulsion is still speculative. Perhaps, a useful long-range communications device could be devised using tachyons. More importantly, if tachyons were discovered, it would give real hope that someday faster-than-light travel might be possible

\section{ACKNOWLEDGMENTS}

We wish to acknowledge very useful discussions with G.W. Ford, W.E. Hazen, J.C. Lee, R.R. Lewis, and J.C.

Zorn. The theoretical analysis in preparation for this experiment has been funded by the National Science Foundation Grant PHY-9731861 and the University of Michigan.

\section{REFERENCES}

Adkins, G.S., and Shiferaw, Y. "Two-Loop Corrections to the Orthopositronium and Parapositronium Decay Rates Due to Vacuum Polarization," Phys. Rev. A 52, 2442 (1995)B.

Adkins, G.S., and Lymberopoulos, M., "Light-by-Light Scattering Contribution to the Decay Rate of Orthopositronium," Phys. Rev. A. 51, R875 (1995)A, "Contribution of Light-by-Light Scattering to the Orders $0\left(m \alpha^{8}\right)$ and $0\left(m \alpha^{8} \ln \alpha\right)$ Orthopositronium Decay Rate," Phys. Rev. A 51, 2908 (1995)A.

Adkins, G.S., and Sapirstein, J. private communication, 10/1998. 
Adkins, G.S. Analytic Evaluation of the Orthopositronium-to-Three-Photon Decay Amplitudes to One-Loop Order" Phys. Rev. Lett. 76, 4903 (1996).

Al-Ramadhan, A.H., and Gidley, D.W. "New Precision Measurement of the Decay Rate of Singlet Positronium." Phys. Rev. Lett. 72, 1632 (1994).

Asai, S., Orito, S., and Shinohara, N. "New Measurement of the Orthopositronium Decay Rate." Phys. Lett. B 357, 475 (1995).

Baltay, C., Feinberg, G., Yeh, N., and Linsker, R. "Search for Uncharged Faster-than-Light Particles." Phys. Rev. D 1, 759 (1970).

Bartlett, D. F., Soo, D., and White, M.G. "Search for Tachyon Monopoles in Cosmic Rays," Phys. Rev. D. 18, 2253 (1978).

Bhat, P.N. et al., "Search for Tachyons in Extensive Air Showers." J. Phys. G: Nucl. Phys 5, L 13 (1979).

Bilaniuk, O.M, Deshpande, V.K., and Sudarsham, E.C.G. ""Meta" Relativity," Am. J. Phys. 30, 718 (1962).

Burichenko, A.P. "Large contribution to the correction $\sim \alpha^{2}$ to the width of orthopositronium," Yad. Fiz. 56, 123 (1993) Phys. Atom. Nuclei. 56, 640 (1993).

Clay, R.W., and Crouch, P.C. "Possible observation of tachyons associated with extensive air showers," Nature 248, 28 (1974).

Czarnecki, A., Melnikov, K. and Yelkhousky, A., " $\alpha^{2}$ Correction to Parapositronium Decay," Phys. Rev. Lett. 83, 1135 (1999).

Dobroliubov, M.I., Gninenko, S.N., Ignatiev, A.Yu., and Matveev, V.A. "Orthopositronium Lifetime Problem," Int. J. Mod. Phys. A 8, 2859 (1993).

Faustov, R. N., Martynenko, A.P., and Saleev, V.A. "O $\left(\alpha^{2}\right)$ corrections to the orthopositronium decay rate," Phys. Rev. A 51, 4520 (1995).

Feinberg, G. "Possibility of Faster-than-Light Particles," Phys. Rev. 159, 1089 (1967).

Feinberg, G. "Particles that go faster than light," Scientific American 222 No. 2 (Feb), 68 (1970).

Fernandez, E. et al., "Tests of quantum electrodynamics with two-, three-, and four-photon final states from $\mathrm{e}^{+} \mathrm{e}^{-}$annihilations at $\sqrt{\mathrm{s}}=29 \mathrm{GeV}, "$ Phys. Rev. D 35, 1 (1987).

Ford, G.W., Sander, L.M., and Witten, T.A. "Lifetime Effects of Positronium in Powders." Phys. Rev. Lett. 36, 1269 (1976).

Gidley, D.W., Nico, J.S., and Skalsey, M. " Direct Search for Two-Photon Decay Modes of Orthopositronium." Phys. Rev. Lett. 66, 1302(1991).

Glashow, S.L. "Positronium versus the Mirror Universe," Phys. Lett. B 167, 35 (1986).

Gninenko, S.N. " Limit on "disappearance" of orthopositronium in vacuum," Phys. Lett. B. 326, 317 (1994).

Hazen, W.E. et al., "A search for Precursors to Extensive Air Showers," Proc. $14^{\text {th }}$ Inter'al Conf. on Cosmic Rays, Munich, (1975) p. 2485.

Hikasa, K, et al. "Review of Particle Properties," Particle Data Group, Phys. Rev. D 45, S1 (1992).

Hyodo, T. priv. comm. (1997).

Kakimoto, M., Hyodo,T., and Chang, T.B. "Conversion of ortho-positronium in low-density oxygen gas," J. Phys. B: At. Mol. Opt. Phys. 23, 589 (1990).

Kantele, J. , and Vakonen, M. " Corrections for Positon Annihilation In Flight In Nuclear Spectroscopy," Nucl. Instr. Meth. 112, 501 (1973).

Karshenboim, S.G. "New logarithmic contributions in muonium and positronium," JETP 76, 541 (1993).

Kinoshita, T. "Quantum Electrodynamic,s" (World Scientific, Singapore, 1990).

L3 Collab. "Test of QED in $\mathrm{e}^{+} \mathrm{e}^{-} \rightarrow \gamma \gamma$ at LEP." Phys. Lett. B 250, 199 (1990).

Labelle, P., Lepage, G.P., and Magnea, U. "Order $m \alpha^{8}$ Contribution to the Decay Rate of Orthopositronium," Phys. Rev. Lett. 72, 2006 (1994).

Marini, et al., "Experimental limits on quarks, tachyons, and massive particles in cosmic rays," Phys. Rev. D. 26, 1777 (1982).

Martyn, H.U. "Test of QED by High Energy Electron-Positron Collisions," in (Kinoshita, 1990)

Mikaelian, K.O. "Orthopositronium Decay into Axions," Phys. Lett. B 77, 214 (1978).

Milstein, A.I., and Khriplovich, I.B. "Large relativistic corrections to the positronium decay probability," JETP 79, 379 (1994).

Mitsui, T. et al., "Search for Invisible Decay of Orthopositronium." Phys. Rev. Lett. 70, 2265 (1993).

Nico, J.S., Gidley, D.W., Rich, A., and Zitzewitz, P.W. "Precision measurement of the Orthopositronium Decay Rate using the Vacuum Technique," Phys. Rev. Lett. 65, 1344 (1990).

OPAL Collab. "Measurement of the cross sections of the $\mathrm{e}^{+} \mathrm{e}^{-} \rightarrow \gamma \gamma$ and $\mathrm{e}^{+} \mathrm{e}^{-} \rightarrow \gamma \gamma \gamma$ at LEP," Phys. Lett. B 257, 531 (1991).

Prescott, J.R. "Tachyons revisited-comments on a search for Faster-than-Light Particles," J. Phys. G: Nucl. Phys. 2, 261 (1976).

Recami, E. "Classical Tachyons and Possible Applications," Riv Nuovo Cim. 9, 1 (1986).

Rich, A. "Recent Experimental Advances in Positronium Research," Rev. Mod. Phys. 53, 127 (1981).

Rolnick, W.B. "Implications of causality for Faster-than-Light Matter," Phys. Rev. 183, 1105 (1969).

Skalsey, M. "Exotic decays of Positronium and C-Odd bosons," Mater. Sci. Forum 255-7, 209 (1997)A.

Skalsey, M., and Conti, R.S. "Search for very weakly interacting, short-lived, C-odd bosons and the orthopositronium decay rate problem," Phys. Rev. A 55, 984 (1997)B.

Skalsey, M., Engbrecht, J.J., Bithell, R.K., Vallery, R.S., and Gidley, D.W. "Thermalization of Positronium in Gases," Phys. Rev. Lett. 80, 3727 (1998).

Smith, G.R., and Standil, S. "Search for tachyons preceding cosmic ray extensive air showers of energy $\geq 10^{14} \mathrm{eV}$," Can. J. Phys. 55, 1280 (1977).

Westbrook, C.I., Gidley, D.W., Conti, R.S., and Rich, A. "Precision measurement of the orthopositronium vacuum decay rate using the gas technique," Phys. Rev. A 40, 5489 (1989). 